\title{
Pemphigusvulgaris in pregnancy. A rare case report
}

\section{loannis Kokolakis ${ }^{1}$, Fanis Makrigiannakis ${ }^{1}$, Sabine Kruger ${ }^{2}$, Konstantinos Krasagakis², Antonios Makrigiannakis ${ }^{1}$}

'Department of Obstetrics and Gynecology, University Hospital of Heraklion, Heraklion, Greece ${ }^{2}$ Department of Dermatology, University Hospital of Heraklion, Heraklion, Greece

\section{Correspondence}

loannis Kokolakis, email: kokolakisioannis@gmail.com

\section{Abstract}

Pemphigus vulgaris (PV) is an autoimmune, bullous, mucocutaneous and potentially life-threatening disease. During pregnancy the occurrence of PV is exceedingly rare and its condition will become more complicated due to different mother's hormone level and the effect of treatment on both mother and her fetus. PV may be associated with an adverse outcome, such as fetal growth restriction and preterm births. We report a case of a 33-year-old woman who was firstly diagnosed with PV during pregnancy. Treatment of highdoses systemic corticosteroids was instituted to control the dis-ease. She delivered a healthy live preterm baby, appropriate for gestational age with no skin or mucosal lesions or other apparent complications.

Key words: Pemphigus vulgaris; corticosteroids; autoimmune; pregnancy

\section{Introduction}

Pemphigus vulgaris is an extremely rare autoimmune, mucocutaneous blistering disease. There are mainly three types of pemphigus: Pemphigus vulgaris (PV), Pemphi-gus foliaceus (PF), and other variants of pemphigus. PV produces blisters in skin and mucous membranes, while pemphigus foliaceus involves skin only, paraneoplastic pemphigus produces blisters in patients with underlying malignancy ${ }^{1}$. The patho-genesis of pemphigus vulgaris is associated with autoantibodies directed against transmembrane glycoproteins of desmosomes, which causes the formation of Dsg3-depleted desmosomes in PV and loss of cell-cell adhesion in the basal and suprabasal layers of the deeper epidermis, with keratinocytes in the superficial layers of the epi-dermis maintaining their cell adhesion. This leads to intercellular edema with loss of intercellular attachments in basal layer and suprabasal epidermal cells separate from basal cellsto form clefts and blisters ${ }^{2}$.

It usually affects the elderly, between 5th and 6th decade of life and genetics seem to play an important role. Although all races are affected, Jews are more susceptible. It is common in eastern countries like India, Malaysia and China and rare in west ${ }^{3}$. The occurrence of PV in pregnancy is very rare. PV in females may be associated with other autoimmune diseases and infertility 4 . Impact on the fetus may be due to the disease by itself or because of the effect of 
treatment given to the mother 5 . Litera-ture review on pemphigus in pregnancy is limited to 47 cases, reported between 1966 and 2014, with diagnosis before or during pregnancy ${ }^{6}$. The neonatal pemphigus is a very rare complication and there are only 29 reported cases on literature review ${ }^{7}$. Mortality rates associated with PV have decreased to $10 \%-15 \%$ with systemic corticos-teroids from a mortality rate as high as $70 \%$ in the precorticosteroid era ${ }^{8}$.

\section{Case reports}

A 33-year-old albanian national, tertigravida (G:3,P:2) with no known chronic illness or medication use, presented at her 23rd week of pregnancy with widespread blistering dermatitis in the abdomen with associated burning and pruritus and erosions over the buccal mucosa. On examination, there were multiple ruptured blisters in the abdomen, a singleton fetus with cephalic presentation and appropriate weight for the week of gestation and rest of the examination was normal.

Differential diagnoses considered of: 1) Herpes simplex infection 2) Herpes zoster 3) Porphyria 4) Impetigo 5) Toxic epidermal necrolysis 6) Systemic lupus erythematosus 7) Pemphigoid gestationalis 8) Steven Johnson's syndrome 9) Pemphigus vulgaris. Lab tests were done including Tzanck smear for herpes, herpes simplex virus (HSV) antibo-dies, ANA\& ANCA tests, PCR for HSV 1 and 2 and a skin biopsy was performed and sent for histopathology and immunoflourescence. Skin biopsy histollogically revealed su-prabasal acantholysis, and direct immunofluorescence showed diffuse intercellular IgG in the epidermidis and basal intercellular C3, which confirmed the diagnosis of PV (Figure 1-2). In addition, anti-desmoglein I, III antibodies were positive. All lab tests were normal except from the previous mentioned confirmatory tests for PV that were positive.

After discussions with the patient about possible adverse effects to the fetus, treatment of high-doses systemic corticosteroids was instituted to control the disease, in collaboration with dermatologists. Possible alteration of the course of pemphigus due to the hormonal and immunologic changes seen in pregnancy was considered before starting the treatment. Due to the severity of the disease she was hospitalized. Pred-nisolone $0,7 \mathrm{mgr} / \mathrm{kg}$ iv daily was initiated from 23 weeks to 28 weeks of gestation with satisfactory clinical response during the first month. Oral ulcers improved, lesions on the abdomen started healing and began to dry up.

The patient developed gestational diabetes and was treated with long acting insulin. Mother and fetal well-being were monitored closely. Following improvement of symp-toms and with no new-onset clinical-skin manifestations, prednisolone was tapered off to $0.6 \mathrm{mgr} / \mathrm{kg}$ iv daily from 28 weeks to 29 weeks of gestation, $0.5 \mathrm{mgr} / \mathrm{kg}$ iv daily from 29 weeks to 31 weeks of gestation and $0,4 \mathrm{mgr} / \mathrm{kg}$ per os daily from 31 weeks to 34 weeks of gestation, when the mother gave birth. She underwent emergency caesarean delivery (history of 2 cesarian sections) due to premature uterine contractions. She delivered a premature, otherwise healthy newborn boy 2.180 gr and it was noted that the neonate showed no skin or oral-mucosa lesions or anomalies while in the neonatal intensive care unit.

The postpartum status of pemphigus vulgaris in the mother was slightly exacerbated after delivery, with new onset lesions under the breast and abdomen. She also devel-oped endometritis three weeks after delivery and was hospitalised and administered intravenously antibiotics. She is still under follow-up for pemphigus vulgaris, 3 months after delivery.

\section{Discussion}

Pemphigus is expressed by extensively distributed bullae and erosions on the skin and mucosa membranes. Pemphigus vulgaris during pregnancy is ex- 

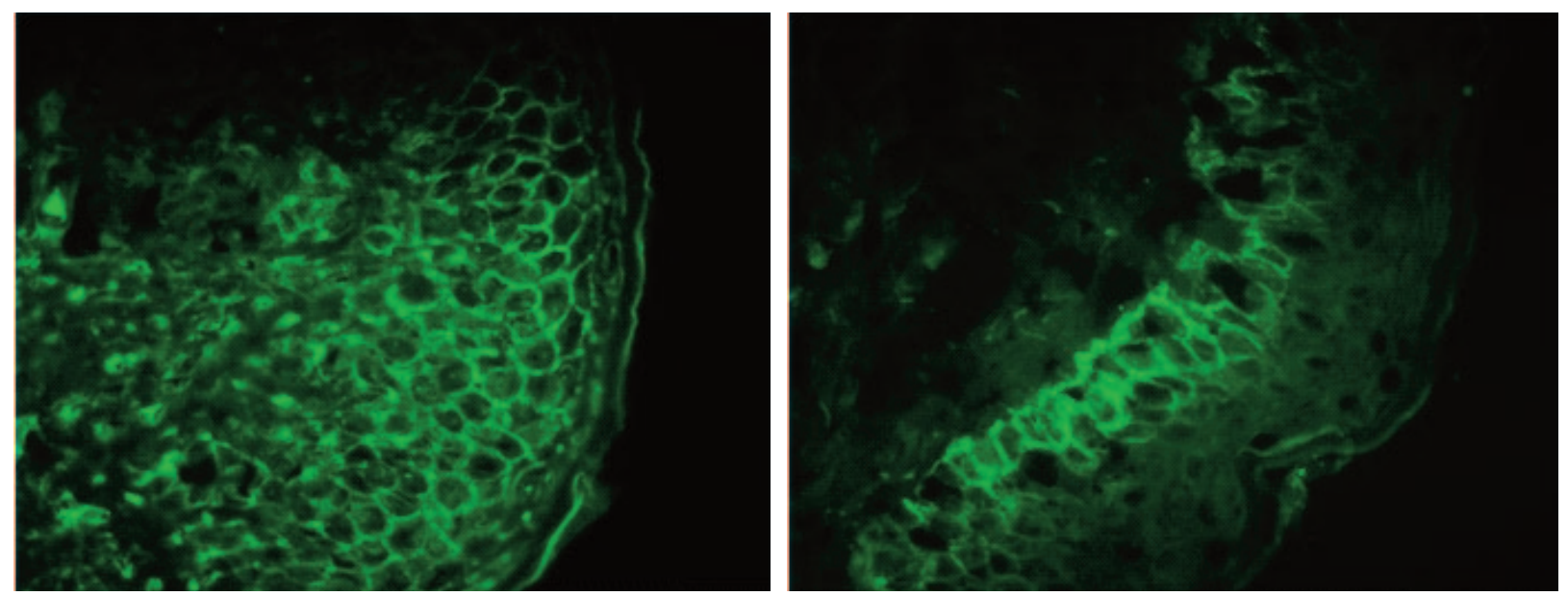

Figure 1,2. Immunofluorescence showed diffuse intercellular IgG in the epidermidis and basal intercellular C3, which confirmed the diagnosis of PV.

tremely rare because it is associated with infertility when it is active ${ }^{4}$.

Pemphigus and pregnancy interact with each other making treatment more complicated for the clinician. The appropriate treatment in pemphigus vulgaris patients during pregnancy is glucocorticoids and it is similar to the treatment without pregnancy ${ }^{9}$. In our case, prednisolone (FDA pregnancy category C) was administered, which can be relatively safely used as immunosuppressive drug during pregnancy as it does not readily cross the placenta and is partially inactivated as it cross the placenta ${ }^{10}$. Prednisolone is safer drug compared with other less used glucocorticoids such as dexamethasone and betamethasone. It should be avoided, if possible, during the first trimester, when risk for defects of the hard palate are highest ${ }^{11,12}$. In our case 6 weeks after treatment, there was remission of the disease with no presence of new clinical-skin manifestations and the disease was slightly aggravated postpartum. The disease is exacerbated most commonly during the first, second trimester, and postpartum, and is stable during the third trimester. This could be due to the increased level of endogenous corticosteroid hormone chorion of the placenta and consequent immunosuppression ${ }^{13}$. In our patient, there was postpartum aggravation of the disease, which is consistent with some studies that report the postpartum flare up of pemphigus vulgaris due to the rapid decrease of corticosteroid hormones levels ${ }^{4}$.

Treatment is often required to control both maternal disease and fetal outcomes. PV may be associated with an adverse outcome, such as fetal growth restriction, intrauterine death, premature delivery, and in approximately $30 \%$ neonatal PV of the newborns ${ }^{14}$. Neonatal pemphigus is caused by the transplacental transmission of antibodies, and only a small amount of immunoglobulin G (IgG) is synthesized by the neonate itself ${ }^{15}$. Poor outcomes have been related with inadequate maternal disease control and higher Dsg antibody titers from maternal serum or umbilical cord blood ${ }^{16}$. In our case, PV was well controlled and the mother gave birth to a premature, otherwise healthy neonate that showed no skin or oral-mucosa lesions or anomalies .

Typical complications observed in these PV patients are skin infections, sepsis, hypoka-lemia, hypoalbuminaemia and Cushing's syndrome due to prolonged steroids usage. She developed postpartum endometritis and skin infections many times and was treated with antibiotics. Our patient is totally treated for 5 months and is still under follow-up. 


\section{Conclusion}

Pemphigus vulgaris in pregnancy is very rare and treatment guidelines have not yet been clarified, thus, management of these cases is individually determined, presenting challenges for the clinician. The good outcome of pregnancies complicated by pemhigus vulgaris is more likely to be obtained from the close collaboration of obstetricians and dermatologists. Current data suggest increased rate of perinatal morbidity and mortality. In summary, pregnancy may have an adverse course, therefore, careful monitoring of the high risk mother and fetus is mandatory.

\section{References}

1. Ruocco E, Wolf R, Ruocco V, Brunetti G, Romano F, Lo Schiavo A. Pemphigus: asso-ciations and management guidelines: facts and controversies. Clin Dermatol. 2013;31:382-390.

2. Joly P, Gilbert D, Thomine E, Zitouni M, Ghohestani R, Delpech A, et al. Identi-fication of a new antibody population directed against a desmosomal plaque anti-gen in Pemphigus and Pemphigus foliaceus. J Invest Dermatol. 1997;108:469-475.

3. Firooz A, Mazhar A, Ahmed AR. Prevalence of autoimmune diseases in the family members of patients with Pemphigus vulgaris. J Am Acad Dermatol 1994; 31 (3 Pt 1): 434-437.

4. Kardos M, Levine D, Gurcan H, et al. Pemphigus vulgaris in pregnancy: analysis of current data on the management and outcomes. Obstet Gynecol Surv.2009;64:739-749.

5. Salzberg KW1, Gero MJ, Ragsdale BD. Pemphigus vulgaris in pregnancy.Cutis. 2014 Oct;94(4):206-9.

6. Lin L., Zeng X., Chen Q. Pemphigus and pregnancy. Analysis and summary of case reports over 49 years. Saudi Med J. 2015 Sep;36(9):1033-8.

7. Daneshpazhooh M, Chams-Davatchi C, Valikhani $\mathrm{M}$, et al. Pemphigus and pregnancy: a 23-year experience. Indian J Dermatol Venereol Leprol
2011;77:534

8. Carson PJ, Hameed A, Ahmed AR. Influence of treatment on clinical course of pem-phigus vulgaris. J Am Acad Dermatol.1996; 34:645-652.

9. Ramsey-Goldman R, Schilling E. Immunosuppressive drug use during pregnancy. Rheum Dis Clin North Am. 1997;23:149-167.

10. van Runnard Heimel PJ, Schobben AF, Huisjes AJ, Franx A, Bruinse HW.The trans-placental passage of prednisolone in pregnancies complicated by early-onset HELLP syndrome. Placenta. 2005 Nov; 26 (10): 842-5.

11. Innes PB. The ultrastructural effects of prednisolone on the mesenchyme of the palatal shelf in the mouse. J Craniofac Genet Dev Biol. 1985;5(3): 287-97.

12. Zawoiski EJ. Effect of L-glutamic acid on glucocorticoid-induced cleft palate in ges-tating albino mice. Toxicol Appl Pharmacol. 1980 Oct;56(1): 23-27.

13. Kalayciyan A, Engin B, Serdaroglu S, Mat C, Aydemir EH, Kotogyan A. A retrospec-tive analysis of patients with pemphigus vulgaris associated with pregnancy. Br J Dermatol. 2002;147:396-397.

14. Meurer M. Pemphigus diseases in children and adolescents. Hautarzt. 2009;60:208-216. German.

15. Bjarnason B, Flosadottir E. Childhood, neonatal, and stillborn Pemphigus vulgaris. Int J Dermatol. 1999;38:680-688.

16. Daneshpazhooh M1, Chams-Davatchi C, Valikhani M, Aghabagheri A, Mortazaviza-deh SM, Barzegari M, Akhyani M, Hallaji Z, Esmaili N, Ghodsi SZ. Pemphigus and pregnancy: a 23-year experience. Indian J Dermatol Venereol Leprol. 2011 Jul-Aug;77(4):534

Received 1-6-2019

Revised 18-6-2019

Accepted 26-6-2019 